\title{
Performance Analysis of IEEE 802.15.4 Transceiver System under Adaptive White Gaussian Channel
}

\author{
Ekhlas Kadhum, Russul Haitham
}

Control and Systems Engineering Department, University of Technology, Iraq

\begin{tabular}{l}
\hline \hline Article Info \\
\hline Article history: \\
Received May 2, 2018 \\
Revised Jul 9, 2018 \\
Accepted Aug 2, 2018 \\
\hline
\end{tabular}

Keyword:

AWGN channel

DSSS

IEEE 802.15.4

OQPSK

Zigbee transceiver

\begin{abstract}
Zigbee technology has been developed for short range wireless sensor networks and it follows IEEE 802.15.4 standard. For such sensors, several considerations should be taken including; low data rate and less design complexity in order to achieve efficient performance considering to the transceiver systems. This research focuses on implementing a digital transceiver system for Zigbee sensor based on IEEE 802.15.4. The system is implemented using offset quadrature phase shift keying (OQPSK) modulation technique with half sine pulse-shaping method. Direct conversion scheme has been used in the design of Zigbee receiver in order to fulfill the requirements mentioned above. System performance is analyzed considering to BER when it encountered adaptive white Gaussian noise (AWGN), besides showing the effect of using direct sequence spread spectrum (DSSS) technique.
\end{abstract}

Copyright () 2018 Institute of Advanced Engineering and Science. All rights reserved.

Corresponding Author:

Russul Haitham,

Control and Systems Engineering Department,

University of Technology,

Baghdad, Iraq.

Email: russulh@gmail.com

\section{INTRODUCTION}

Due to the advances in wireless communications and electronics over the last few years, the development of networks of low-cost, low-power, and multi-functional sensors received an increasing attention. These sensors are small in size and able to sense, process data, and communicate with each other over an RF (radio frequency) channel. A sensor network is designed to detect events or phenomena, collect and process data, and transmit sensed information for the intended users [1], [2].These networks have the potential of interfacing the physical world with the virtual (computing) world in an unprecedented scale and provide practical usefulness in developing a large number of applications, including the protection of civil infrastructures [3], habitat monitoring, precision agriculture, toxic gas detection, supply chain management, and health care [4].

Zigbee is considered one of wireless sensor devices operates under IEEE 802.15.4 standard. This standard has been introduces by Zigbee Alliance [5]. Zigbee standard was created to address the market need for cost-effective, standard-based wireless networking solutions that support low data rates transceivers, low power consumption [6], security, and reliability through wireless personal area networks (WPANs) [7]. Zigbee technology can be worked within three various frequency bands according to the geographical coverage area. In Europe, $868-868.6 \mathrm{MHz}(868 \mathrm{MHz})$ is used, in Northern America 902-928 $\mathrm{MHz}(915$ $\mathrm{MHz}$ ), while the $2.4 \mathrm{GHz}$ is used all over the world, and it depends on unlicensed Industrial, Scientific and Medical(ISM) radio bands [8].

It is known that the wireless medium is influenced by real time conditions such as signals interference, doppler shift, and path loss; where these parameters have the effect of degrading wireless transmission performance [9]. Hence, it is quite important to provide a transceiver system that could be able 
to accommodate with such environments.

One of the main challenges in designing a digital transceiver system is to attain a system with such an accepted performance in real time environments. For such system, bit error rate (BER) is considered to be an important measurement factor that determines the number of bits corrupted during transmission against the total number of transferred bits. Hence, determine the efficiency of the digital system in facing such environments.

This research aims to present a model for Zigbee transceiver system based on IEEE 802.15.4 standard, and specifically for the $2.4 \mathrm{GHZ}$ frequency band. The objectives of this research can be summarized as follows:

1. To implement 2.4 GHZ Zigbee transceiver system based on IEEE 802.15.4. Offset quadrature phase shift keying (OQPSK) modulation technique with half sine wave as a pulse shaping, and direct sequence spread spectrum (DSSS) as a spreading technique are such significant specifications within this standard.

2. To analyze system performance, considering BER, under adaptive white Gaussian noise (AWGN) channel and to compare the situations when the system is with or without DSSS.

\section{ZIGBEE ARCHITECTURE}

Based on open system interconnection (OSI) reference model, Zigbee protocol layers can be divided. There are some advantages related with this division. Firstly, whenever protocol changes over time, it is possible to apply the required modification or replacement to the layers affected by that change rather than altering the whole protocol. Secondly, if there are any enhancements to be done for developing an application, it can be attained from a third party independently from the lower layers. In other words, the changes are applied on the application layer only [10]. IEEE 802.15.4 standard defines specifications of the two bottom layers (PHY and MAC layer). The remaining upper layers of Zigbee protocol (network, security, and application profile layer) are defined by Zigbee Alliance [11].

Zigbee PHY layer is responsible for performing spreading, modulation, demodulation and despreading functions [12]. In order to reduce the impact of noise from adjacent networks, direct sequence spreading sequence (DSSS) is used to increase the frequency of the signal hence increasing its power [13]. OQPSK modulation technique and DSSS spreading technique are used in $2.4 \mathrm{GHZ}$ band; where each 4-bit symbol is mapped into a 32 chip PN sequence. In the $915 \mathrm{MHz}$ and $868 \mathrm{MHz}$ bands, the Binary Phase Shift Keying (BPSK) modulation technique is used besides mapping each one-bit symbol into a 15 chip PN sequence [14],[15].

OQPSK, also called Staggered QPSK (SQPSK), is a modified version of QPSK. In this technique, the carrier wave permits to send the signal for four orthogonal phases. The benefit behind this modification is to delay the signal by a half cycle so as to avoid possible change in signal phase [16]-[18]. OQPSK modulation equitation could be written as follows [19]:

$$
s_{i}(t)=\mathrm{A} \cos \left(2 \pi f_{c} t+\theta_{i}\right), \quad 0 \leq t \leq \mathrm{T}
$$

Where:

$$
\theta_{i}=\frac{(2 i-1) \pi}{4}
$$

The carrier phase can take one of the four spaced values: $\frac{\pi}{4}, \frac{3 \pi}{4}, \frac{5 \pi}{4}$, and $\frac{7 \pi}{4}$. Equation (1) can be further written as:

$$
\begin{aligned}
& s_{i}(t)=\mathrm{A} \cos \theta_{i} \cos 2 \pi f_{c} t-\mathrm{A} \sin \theta_{i} \sin 2 \pi f_{c} t \\
& s_{i}(t)=s_{i 1} \phi_{1}(t)+s_{i 2} \phi_{2}(t)
\end{aligned}
$$

Where:

$$
\phi_{1}(t)=\sqrt{\frac{2}{\mathrm{~T}}} \cos 2 \pi f_{c} t, 0 \leq t \leq T
$$




$$
\begin{aligned}
& \phi_{2}(t)=-\sqrt{\frac{2}{\mathrm{~T}}} \cos 2 \pi f_{c} t \\
& s_{i 1}=\sqrt{\mathrm{E}} \cos \theta_{i} \\
& s_{i 2}=\sqrt{\mathrm{E}} \sin \theta_{i} \\
& \theta_{i}=\tan ^{-1} \frac{s_{i 2}}{s_{i 1}}
\end{aligned}
$$

As a result, OQPSK modulation signal can be expressed as:

$$
s(t)=\frac{\mathrm{A}}{\sqrt{2}} \mathrm{I}(t) \cos 2 \pi f_{c} t-\frac{\mathrm{A}}{\sqrt{2}} Q(t) \sin 2 \pi f_{c} t,-\infty<t<\infty
$$

where $f_{c}$ represents the frequency of carrier wave.

In order to measure the performance of digital modulation scheme, two terms have to be considered; its power efficiency and bandwidth efficiency. Power efficiency defines the capability of a modulation technique to maintain the fidelity of the digital message at low levels of power. In a digital communication system, an increasing in signal power leads to an increasing in noise immunity. However, the type of modulation employed determines the possible amount by which the signal power is increased (i.e., an accepted bit error probability) to get a certain level of fidelity. The power efficiency (also called energy efficiency) is defined as the ratio between the signal power per bit and noise power spectral density $E_{b} / N_{o}$ necessary for the receiver input for a certain value of error probability (say $10^{-4}$ ) [20]. BER can be defined as the total number of bits corrupted during transmission to the overall number of bits transferred over a communication channel and it can be calculated as [21]:

$$
B E R=\frac{(\text { Number of transferred bits) }}{\text { (Overall number of bits })}
$$

Furthermore, the ratio of transmitted signal power to the power of noise represents the Signal-to-Noise Ratio (SNR) and it is measured in decibel (dB). SNR can be expressed as [22]:

$$
S N R=10 \log _{10} \frac{(\text { Signal Power })}{\text { (Noise Power })}
$$

For OQPSK, the total probability of error per bit is [11]:

$$
P_{e}=Q(\sqrt{2 \cdot S N R}
$$

where $\mathrm{Q}$ represents the function $\mathrm{Q}(\mathrm{x})$ used to calculate the area under the tail of Gaussian probability distribution function (pdf) and it is calculated as [22]:

$$
\mathrm{Q}(\mathrm{x})=\frac{1}{\sqrt{2 \pi}} \int_{\mathrm{x}}^{\infty} \mathrm{e}^{-\left(\mathrm{t}^{2} / 2\right)} \mathrm{dt}
$$

Form equation (13), it is clear that any increasing in SNR leads to decrease in BER.

\section{PROPOSED SYSTEM}

Features shown in Table 1 are considered for implementing the Zigbee transceiver system. For the transmitter part, the main components including; bit-to-symbol and symbol to chip mapping, serial to parallel conversion, baseband waveform shaping and modulation are depicted. Furthermore, the conversion from RF 
to baseband signal, in addition to D/A conversion, parallel to serial conversion and despreading process are all depicted in the receiver part. Figure 1 and Figure 2 illustrate the basic elements of the proposed transmitter and receiver models. Steps showing below describe the design procedure in more details.

Table 1. Specifications of IEEE 802.15.4 for 2.4 GHZ Zigbee (PHY) layer [9]

\begin{tabular}{lc}
\hline \multicolumn{1}{c}{ Features } & Value \\
\hline Data Rate & $250 \mathrm{kbps}$ \\
Frequency of Operation & $2.4 \mathrm{GHz}$ \\
Number of Channels & 16 \\
Channel Spacing & $5 \mathrm{MHZ}$ \\
Chip Rate & $2 \mathrm{Mbps}$ \\
Pulse Shaping & Half Sine \\
Spreading Technique & DSSS \\
Modulation Technique & OQPSK \\
\hline
\end{tabular}

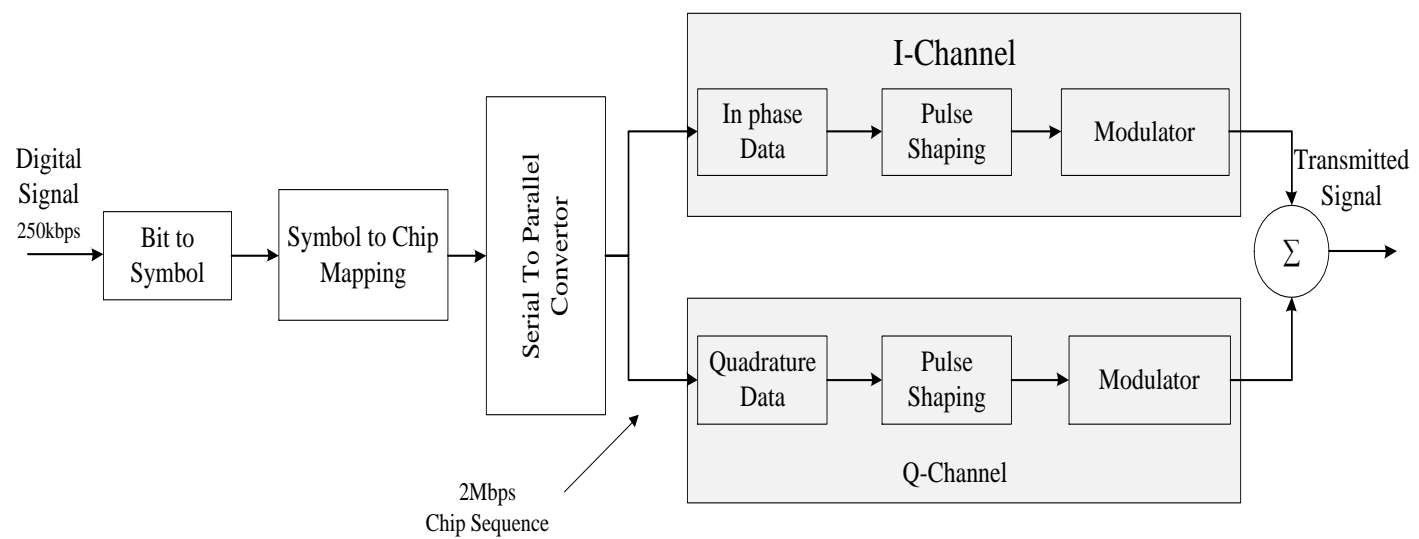

Figure 1. Transmitter model

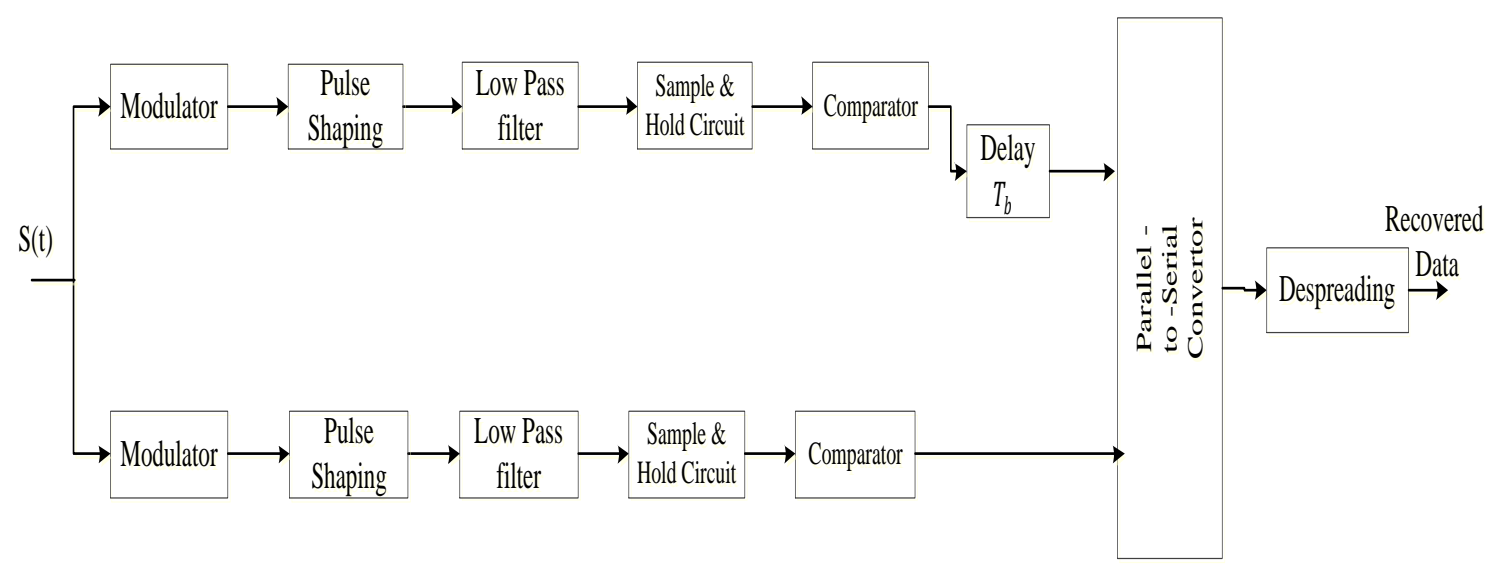

Figure 2. Receiver model

Step1: Preparing the input bit stream: This step includes applying mapping process of bit to symbol and symbol to chip. At first, the input data stream must be prepared to meet IEEE 802.15.4 specifications for data rate and chip rate. Pseudo-random noise (PN) chip sequence is used to construct a direct sequence spread spectrum (DSSS). The reason behind the use of this technique is to increase the frequency of input data stream to $2 \mathrm{Mbps}$ which in turn might improve transmitter performance in multipath environment. It is also used to achieve an improvement in signal to noise ratio (SNR) throughout increasing resistance of the system towards anticipated or accidental jamming 9][. 
Step2: Serial to parallel conversion: Prior to modulation process, the previous coded data are separated into In-phase and Quadrature data. Even and odd clock pulses are generated using JK-flip flop in toggle state; where the positive output $(\mathrm{Q})$ is used as a source of clock pulses for the first D-flip flop to produce the In-phase data, while the negative output is fed to the second D-flip flop producing Quadrate data. Figure 3 depicts the process of serial to parallel conversion.

Step 3: Modulation Process: OQPSK modulation is the same as QPSK except the Q-channel is delayed by $T / 2$ seconds. Although the power density and error performance are the same in the two techniques, OQPSK produces a maximum phase change of $\pi / 2$ in contrast to QPSK which provides a phase shift of $\pi$ [23]. The output of In-phase and Quadrature data is firstly multiplied by half sine wave to perform pulse shaping. The purpose behind the use of pulse shaping (also called pre-modulating filter) is to make the transmitted signal spectrum getting matched with channel bandwidth, hence; avoiding multipath and intersymbol interference (ISI) effects [9]. half sine pulse signal can be represented as [24]:

$$
\mathrm{p}(\mathrm{t})=\sin \frac{\pi \mathrm{t}}{2 \mathrm{~T}_{\mathrm{c}}}
$$

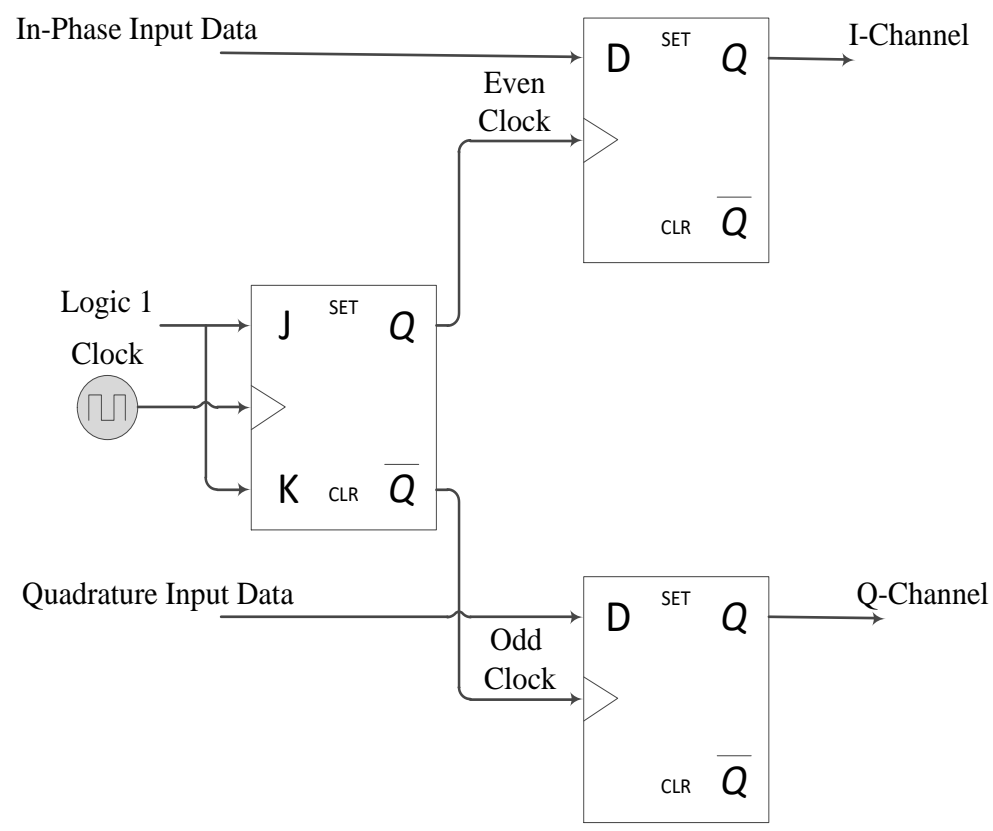

Figure 3. Serial to parallel conversion

Next, the I-channel data is multiplied by a cosine wave of $2.4 \mathrm{GHZ}$ and the Q-channel data is multiplied by a sine wave of the same frequency. The final modulated transmitted signal $s(t)$ is obtained by adding the output of I-channel and Q-channel. At the receiver, the input represents the received transmitted signal after passing through the communication channel. The following steps describe the design procedure of the receiver model:

Step1: RF to Baseband conversion: at first, the demodulation process is performed by multiplying the In-phase data at the receiver side by cosine carrier signal; while the Quadrature data is multiplied by sine carrier signal to produce I-channel and Q-channel modulated signals. The resultant signals are then pulseshaped by half sine pulse shaping signals, similarly as done in the transmitter side. In order to extract the noise produced by the communication channel and to get the right version of the transmitted signal; an analog low pass filter is used with certain parameters.

Step2: A/D conversion: since the produced filtered signals are in analog format, an analog to digital (A/D) convertor is used to generate the digital signal. Zero order hold circuit followed by a comparator is used for this purpose.

Step3: Parallel to serial Conversion: the In-phase and Quadrature data are combined together using a parallel to serial convertor to produce serial data. 
Step4: Despreading process: similarly, as performed with the transmitter side, the resultant serial data is despreaded using the same PN sequence and chip rate specified by the standard. To overcome the delay that might occur, a delay element could be added after the PN sequence to ensure its synchronization with that at the transmitter side.

\section{CHANNEL MODEL}

In order to analyze system performance against the real-time environments, a communication channel has been equipped between the transmitter and receiver model. In this research, Adaptive White Gaussian Noise (AWGN) channel model is used. AWGN channel is considered to be the simplest noise model used by various communication channels and has random processes behavior that exists in nature [23]. For AWGN, the transmitted signal subjected to noise effect $r(t)$ can be expressed as:

$$
r(t)=s(t)+n(t)
$$

where $s(t)$ is the modulated transmitted signal, and $n(t)$ is the noise added by AWGN channel. In this research, as shown in Figure 4, AWGN channel is added between the transmitter and receiver models with a variable range of SNR values.

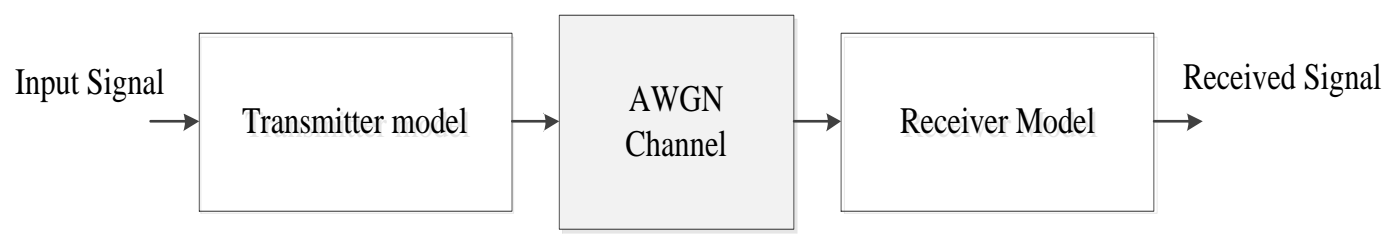

Figure 4. The proposed transceiver system with AWGN channel

\section{RESULTS AND SIMULATION ANALYSIS}

Zigbee transceiver system has been implemented based on IEEE 802.15.4 specifications for 2.4 GHZ frequency band assuming the input signal is in a binary format. Figure 5 shows the general structure of system implementation, which includes the transmitter, receiver, and BER calculator.

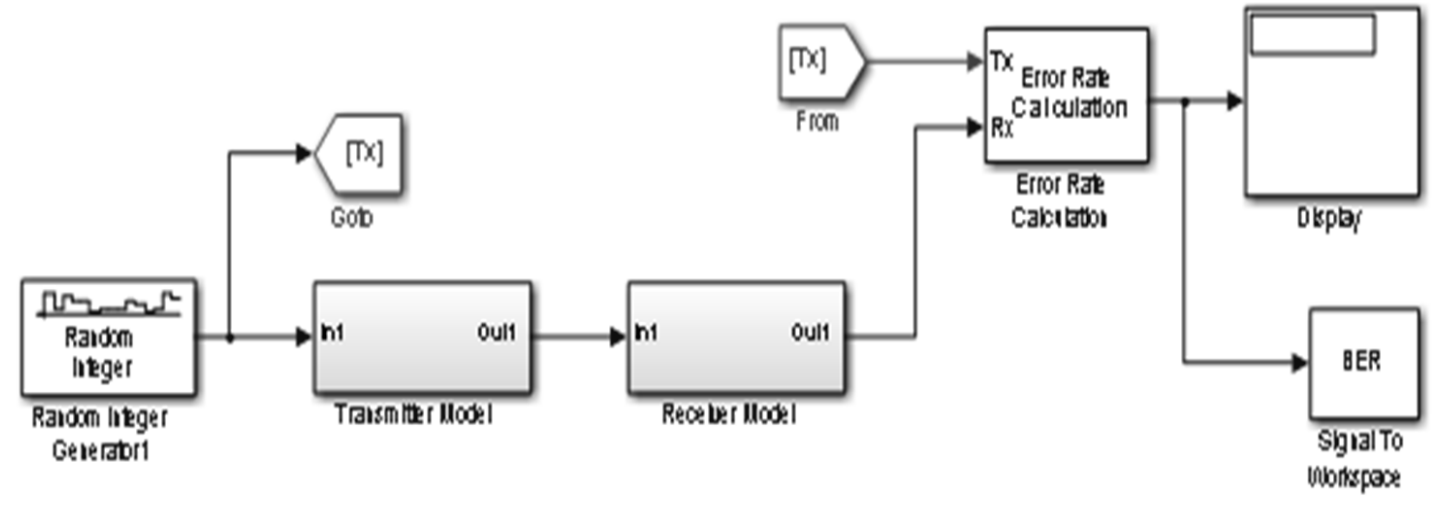

Figure 5. General structure of the proposed transceiver system

An input bit stream has been produced by a Random Integer Generator block with data rate of 250 kbps; i.e. sampling time is $4 \mathrm{e}^{-6} \mathrm{~s}$, and as shown in Figure 6. 


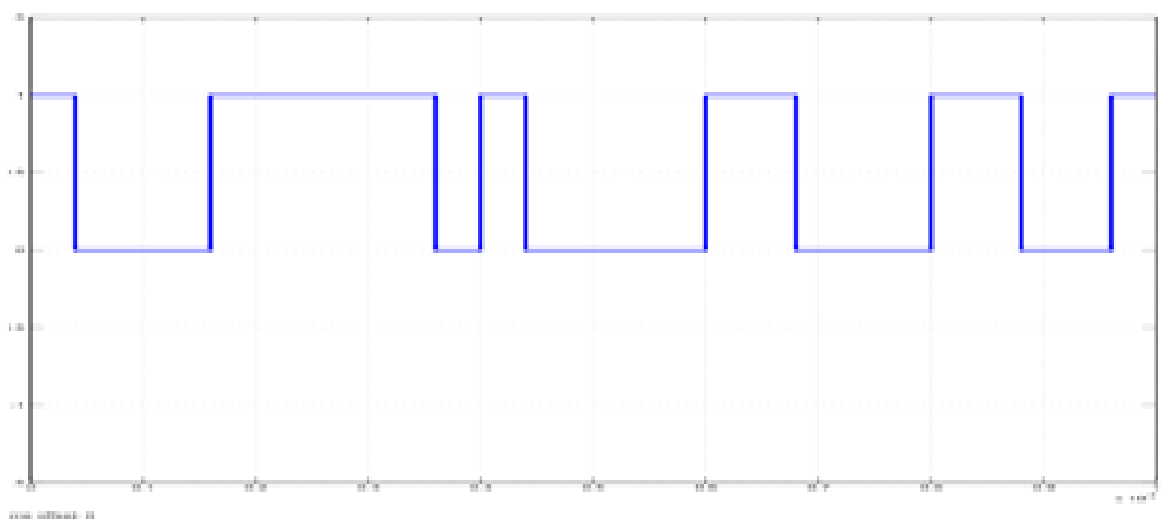

Figure 6. Input data stream

The transmitter and receiver models have been implemented using fundamental components within MATLAB/Simulink to show the possibility in designing radio transmitter and receiver system with complex modulation technique and with appropriate pulse shaping method in reliable and cost-effective way. After generating the input sequence, it has been multiplied by a PN sequence of 32 chips and chip rate of $2 \mathrm{Mbps}$ to produce a DSSS signal. Signal waveforms of input sequence and PN sequence Generator after NRZ conversion are depicted in Figure 7 and Figure 8 respectively, while Figure 9 shows the final signal after spreading.

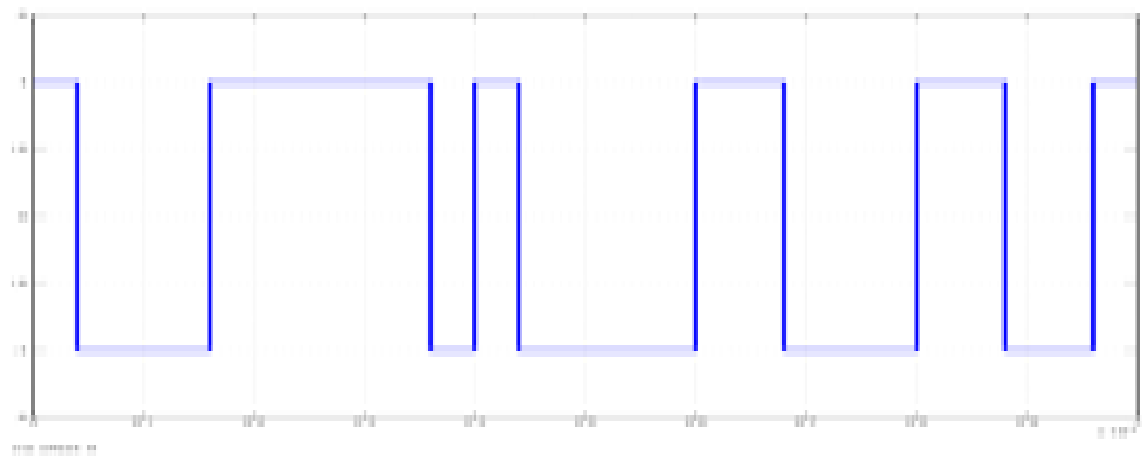

Figure 7. Bipolar input sequence

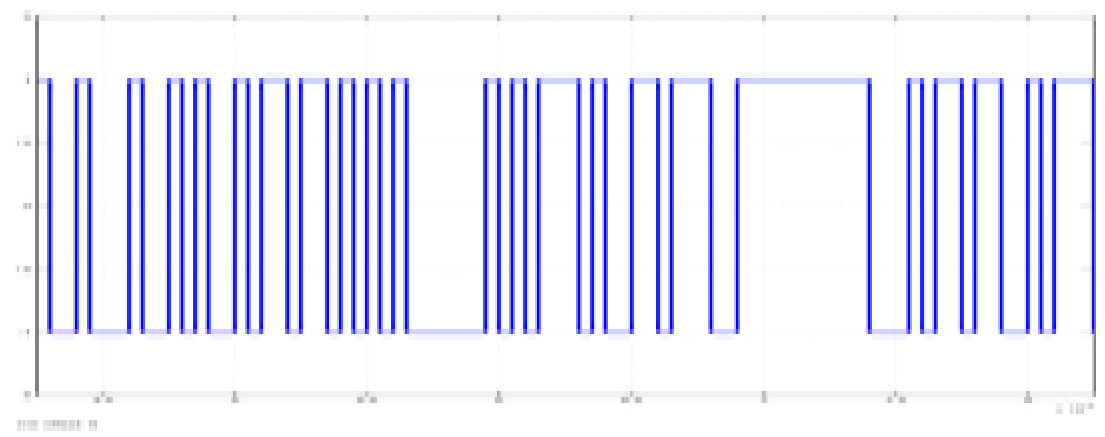

Figure 8. PN Sequence Generator Output Signal 


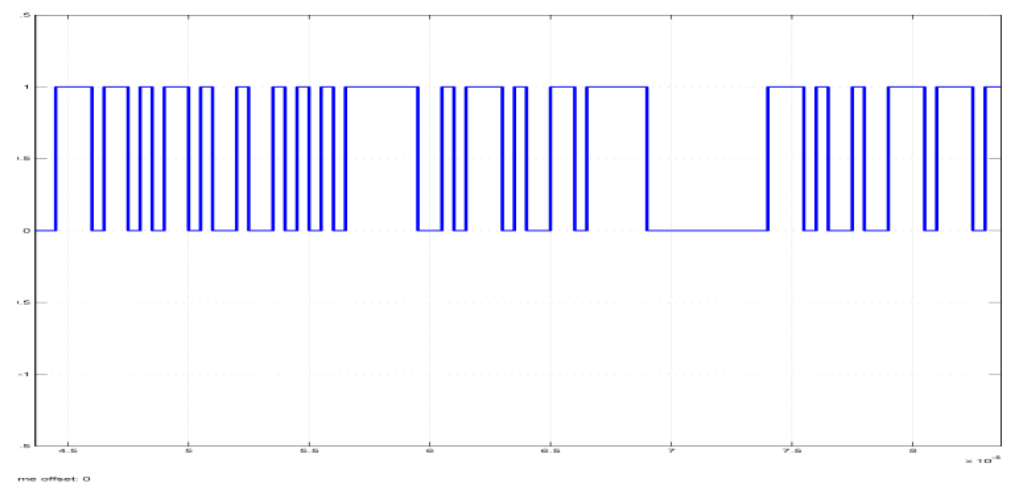

Figure 9. Input sequence after applying DSSS technique

The resulted signal has been divided into even and odd part to produce the In-phase and Quadrature data using serial-to-parallel convertor. The serial to parallel convertor has been constructed using a J-K flip flop and two D flip flops with clock frequency of $16 \mathrm{MHZ}$. since the OQPSK modulation scheme implies that the Quadrature data have to be shifted by one bit period, an extra D flip flop has been used as a delay element. Figure 10 shows the odd and even clock pulses produced by J-K flip flop, while the final In-phase and Quadrature data are shown in Figure 11.

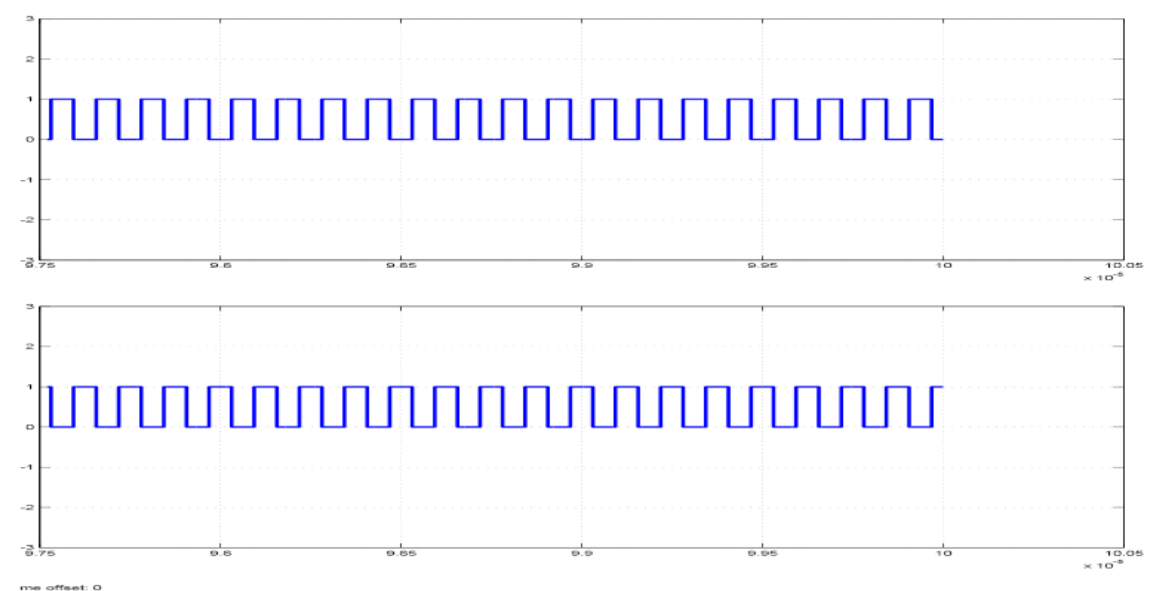

Figure 10. Clock pulses of even and odd data

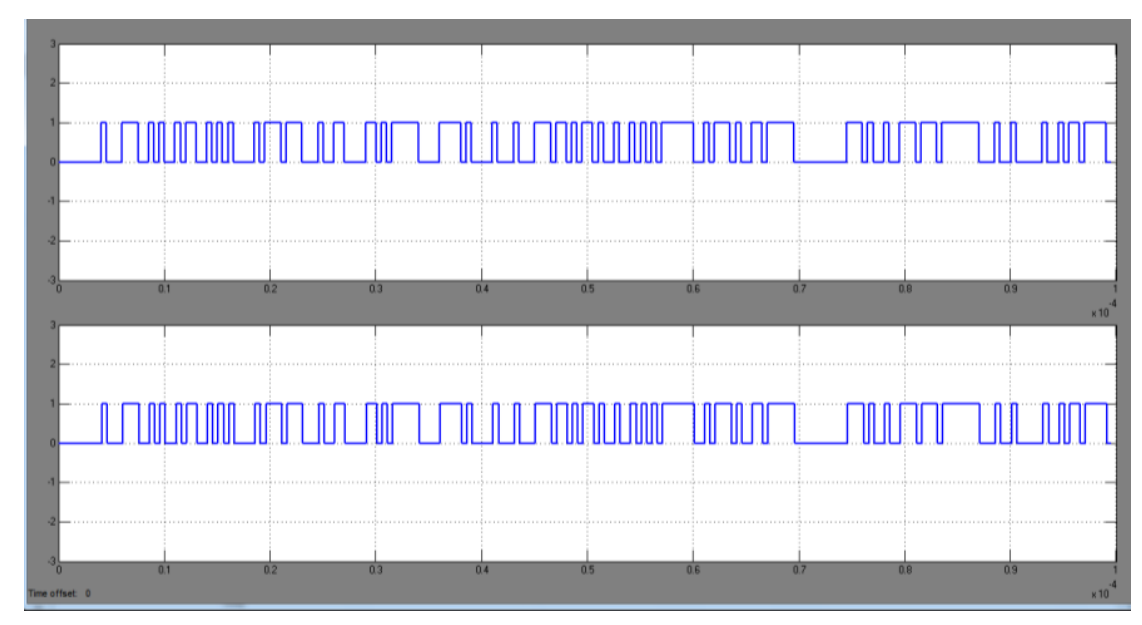

Figure 11. In-Phase and quadrature data 
The output data from In-phase and Quadrature channel have been converted into NRZ format and then multiplied by a half-sine wave at $2 \mathrm{MHZ}$ which has been produced by multiplying $0.5 \mathrm{MHZ}$ pulse wave by a sine wave of $0.5 \mathrm{MHZ}$. Figures 12 and 13 show the generation of half -sine pulses and the resultant output of each channel.

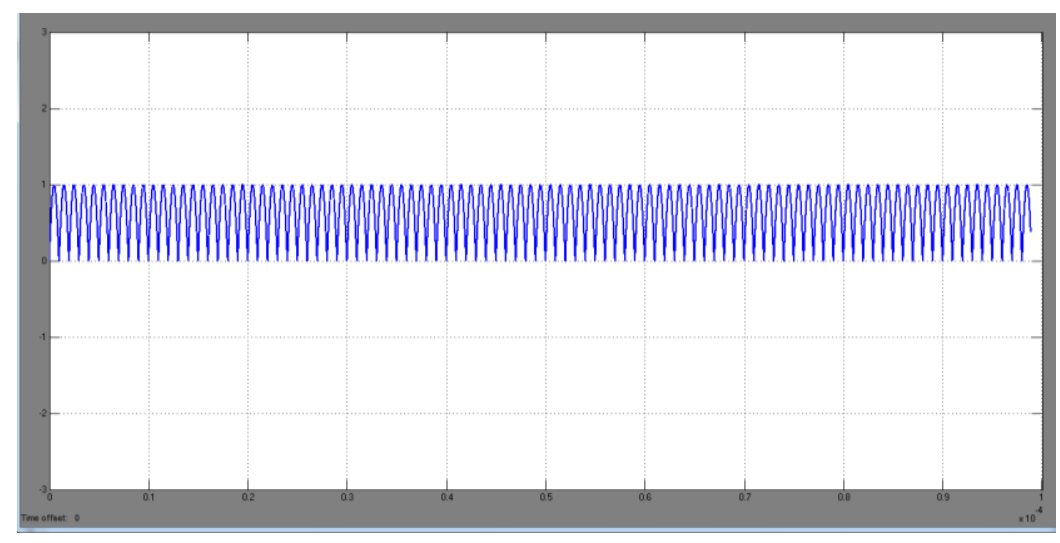

Figure 12. Half sine signal waveform

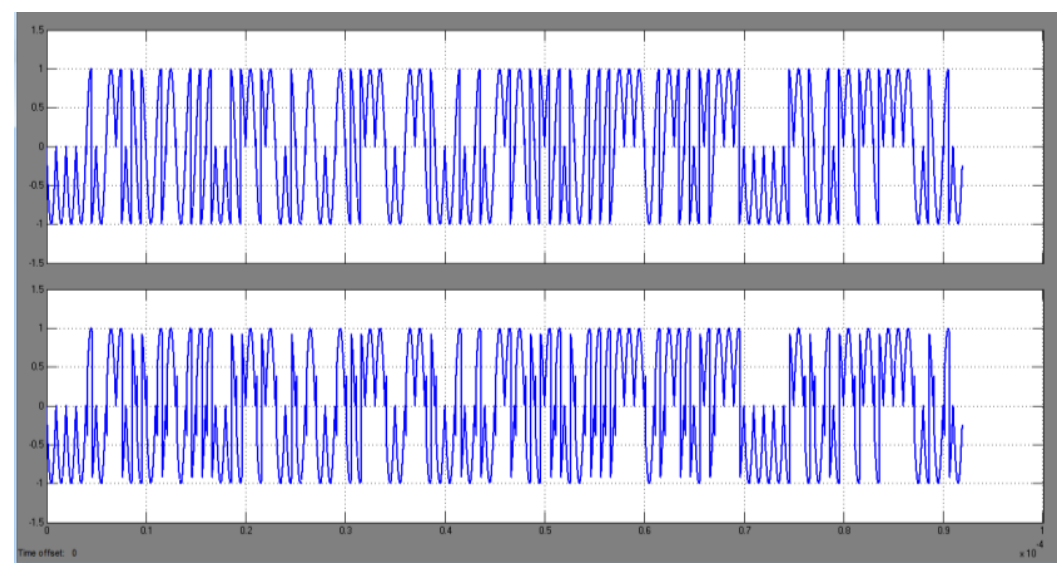

Figure 13. In-phase and quadrature data after half sine pulse shaping

Next, according to equation 10, modulation process has been performed by multiplying the In-phase data by sine wave carrier frequency of $2.4 \mathrm{GHz}$, while the data of Quadrature has been multiplied by orthogonal cosine wave at the same frequency. The final modulated signal has been produced by simply adding the resultant I-channel and Q-channel data and as shown in Figure 14 and Figure 15.

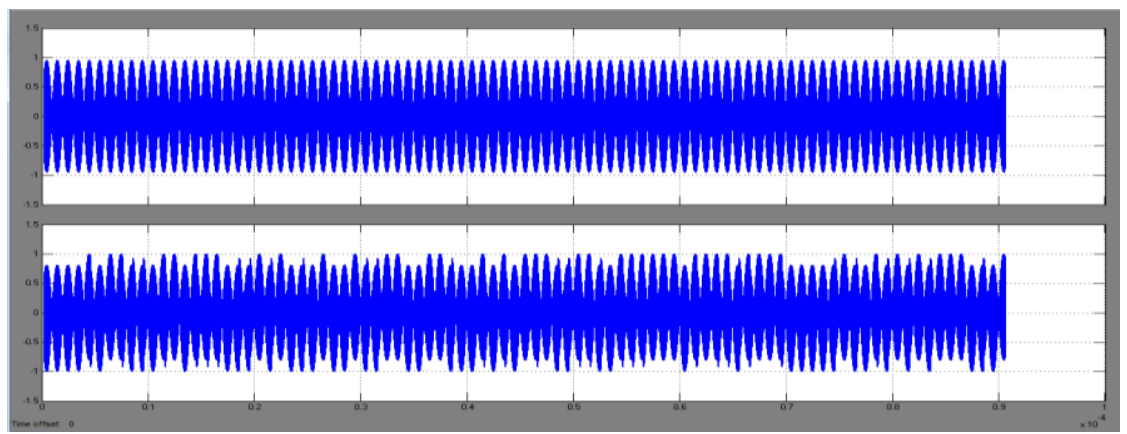

Figure 14. In-phase and quadrature data after modulation process 


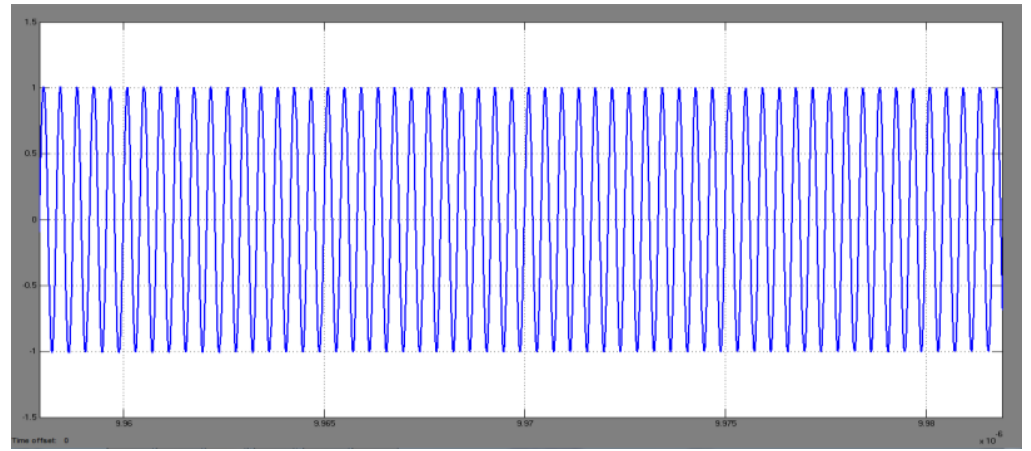

Figure 15. Resultant transmitted signal

At the receiver side, the first step was to convert the received RF signal into a baseband waveform by firstly performing demodulation process through multiplying the In-phase data by sine wave carrier signal and the Quadrature data by cosine wave carrier signal at $2.4 \mathrm{GHZ}$. Then, half sine pulse shaping has been performed, similarly as done with the transmitter side, by multiplying the generated demodulated data by half sine wave of $1 \mathrm{MHZ}$. Finally, an $8^{\text {th }}$ order analog Butterworth low pass filter at $16 \mathrm{MHZ}$ has been used to recover the original data. In sampling and thresholding process, a circuit consists of a zero order hold at sampling time of $6.25 e^{-8} \mathrm{~s}$ and a comparator has been used to implement ADC function. Then, in order to despread the final digital signal, the In-phase and Quadrature data was firstly combined together through parallel-to-serial conversion process, and this was done using a switch at clock frequency of $2 \mathrm{MHZ}$. Eventually, despreading process has been performed by multiplying PN sequence generator of $2 \mathrm{Mbps}$ chip rate by the last serial signal. For synchronization purpose, a sufficient amount of delay has been added after the PN sequence at the receiver side. Figures 16 to 20 demonstrate signal waveforms at different stages of the proposed receiver model, and when the transceiver system passed through AWGN channel model. Data has been successfully recovered as shown in Figure 21.

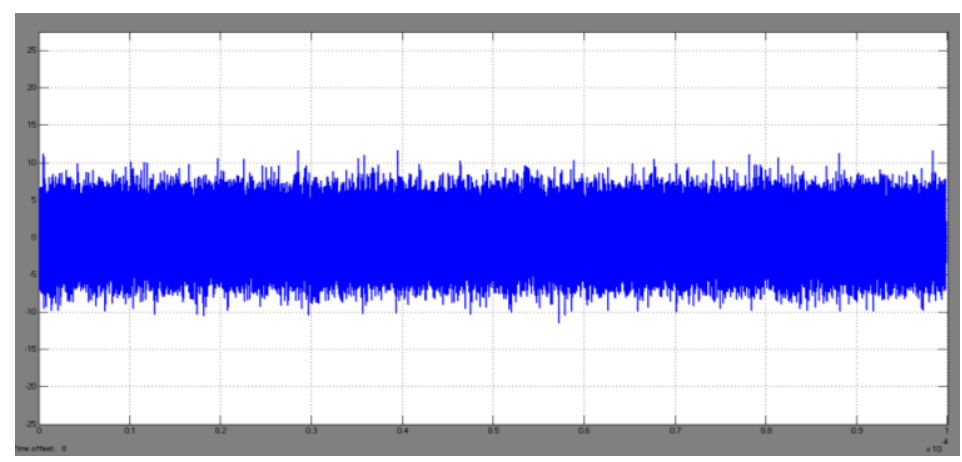

Figure 16. AWGN channel model received signal

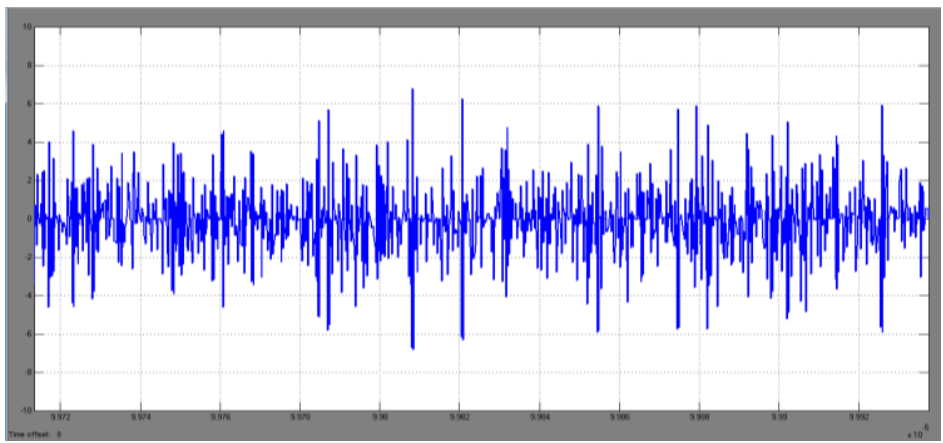

Figure 17. Demodulated In-phase received data 


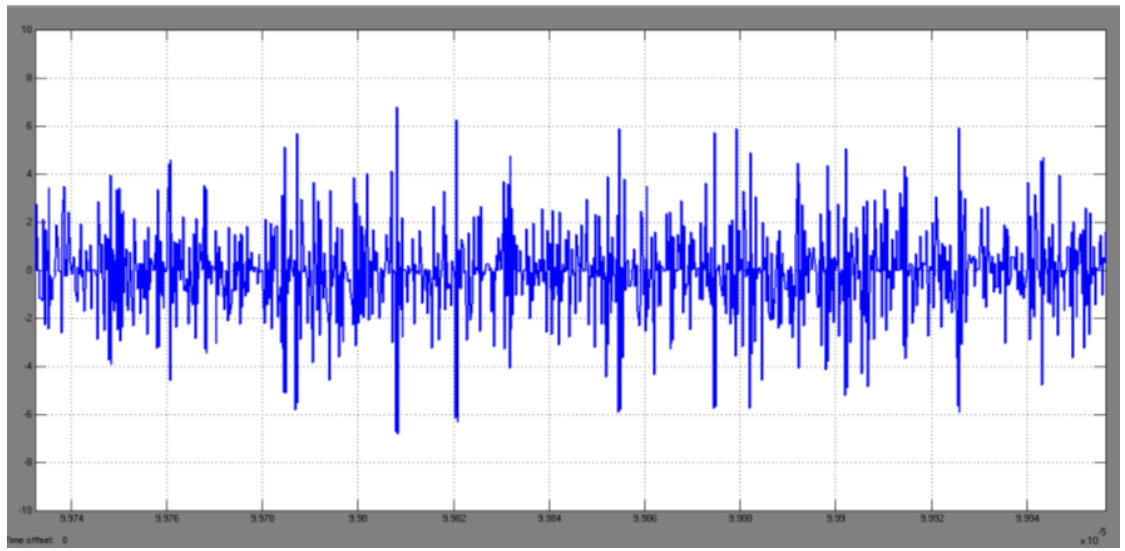

Figure 18. Pulse-shaped In-phase received data

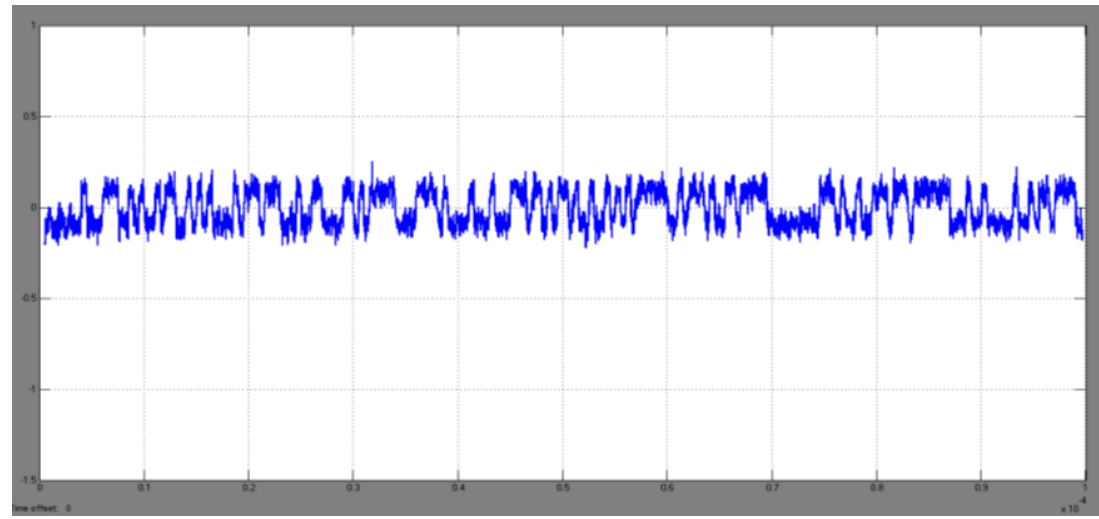

Figure 19. Low pass filter output for In-phase data

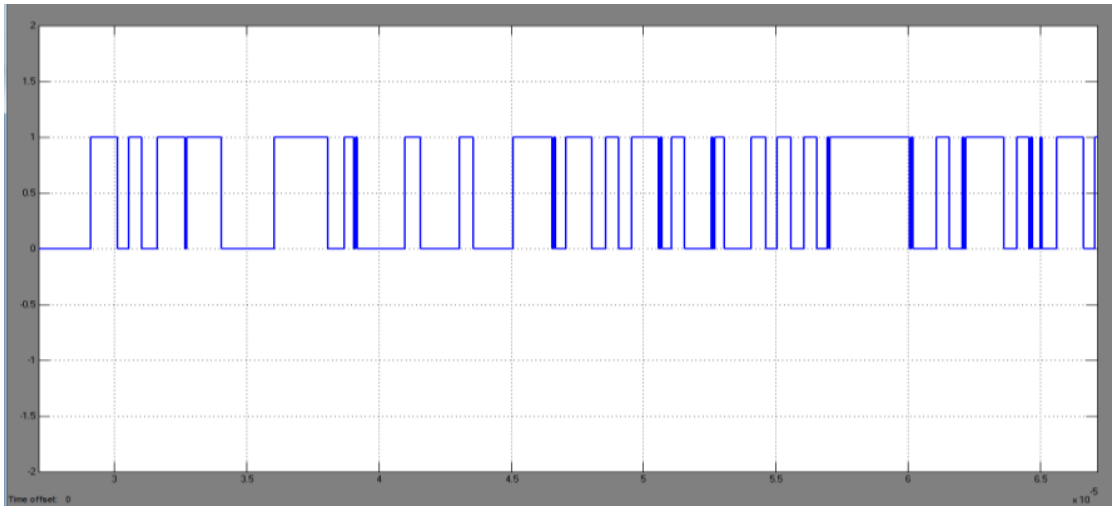

Figure 20. In-phase data compactor output

In order to analyze the proposed system performance, BER VS $E_{b} / N_{o}$ has been measured in presence of AWGN channel. In addition, the effect of DSSS has been taken into consideration. Bit Error Rate Analysis Tool (BERTool) application in MATLAB software has been used to compute the BER as a function of signal-to-noise ratio (SNR) (or $E_{b} / N_{o}$ ). It analyzes performance either with Monte-Carlo simulations of MATLAB functions and Simulink models or with theoretical closed-form expressions for selected types of communication systems. $E_{b} / N_{o}$ values range between $-8 \mathrm{~dB}$ to $4 \mathrm{~dB}$ has been selected to calculate the possible BER values. 


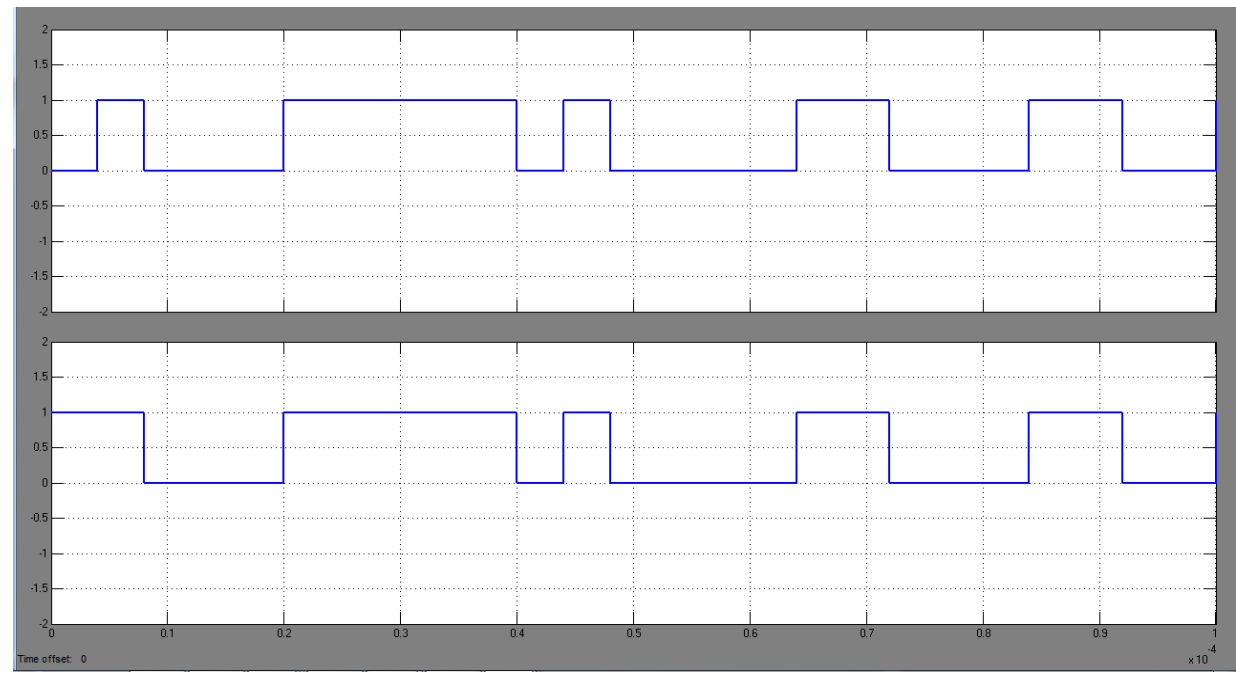

Figure 21. Input and output data of the proposed transceiver system

Since AWGN channel block in MATLAB/Simulink supports only discrete complex input and output signals; it is necessary for both of transmitter output and receiver input signals to be firstly converted into discrete form before they going are fed through the channel, and this has been done using two zero order hold blocks running at sample time of $5 \mathrm{e}^{-10} \mathrm{~s}$. The sample period for AWGN channel was set to be $6.25 \mathrm{e}^{-8} \mathrm{~s}$.

The performance of the proposed Zigbee transceiver System has been analyzed when it passed through AWGN channel; where BER has been compared when the system was with or without DSSS. Figure 22 depicts BER curve for the system with AWGN Channel. According to this curve, any increasing in $E_{b} / N_{o}$ values led to better BER performance. Furthermore, it is observed that the system without DSSS acted better than theoretical OQPSK. For instance, when $E_{b} / N_{o}$ was equal to $2 \mathrm{~dB}$, BER was 0.03 theoretically; while it was equal to $10^{-2}$ without DSSS, and $10^{-3}$ with DSSS.

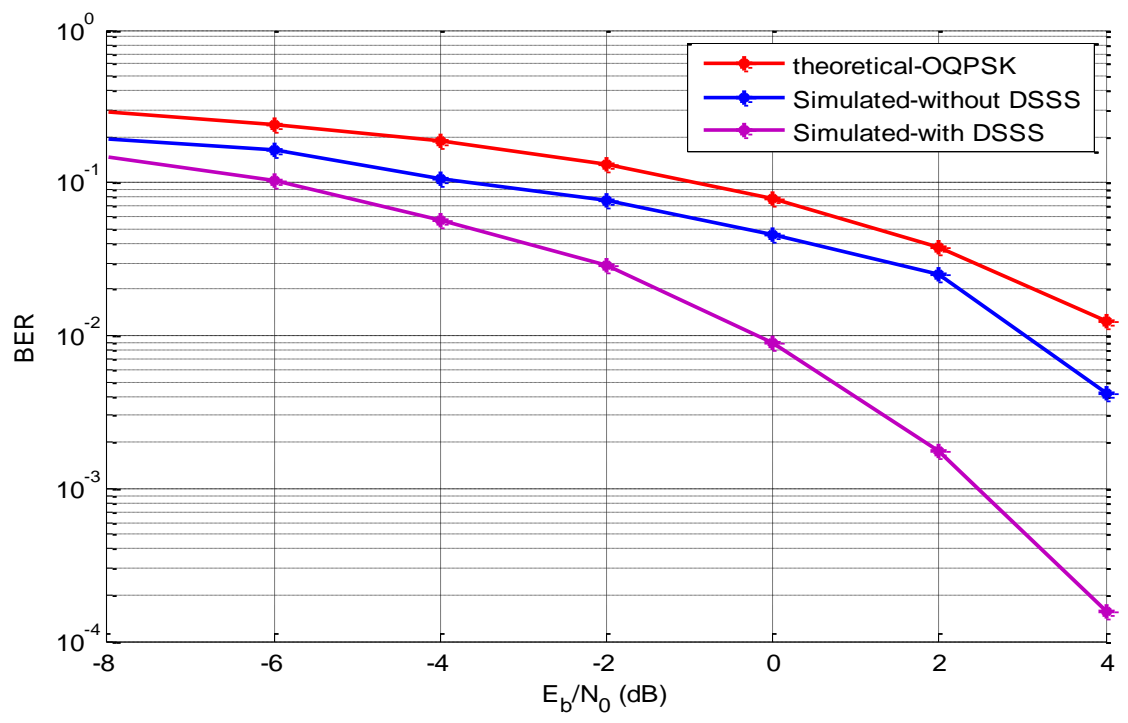

Figure 22. BER performance with AWGN channel model

From the previous work results, it was shown that the proposed zigbee transceiver system has achieved a more desirable performance considering to BER when it was experienced by AWGN channel. The use of DSSS technique has the benefit of further reducing the interference effects. In addition, the use of direct conversion scheme in the receiver design has fulfilled the requirements of expected designing i.e. low 
cost and low power consumption. Also, the proposed transceiver system shows better performance for $E_{b} / N_{o}$ equals to $4 \mathrm{~dB}$, as an example, comparing with that suggested by [9].

\section{CONCLUSIONS}

Digital transceiver system for 2.4 GHZ Zigbee sensor device based IEEE 802.15.4 has been implemented. Different points have been considered in determining the performance of the proposed system by examining their effects in BER measurement. Simulation results have shown that the proposed Zigbee transceiver has a better performance in presence of DSSS than in the case without. For $E_{b} / N o$ values range between $-8 \mathrm{~dB}$ to $4 \mathrm{~dB}$, the BER was ranging between $10^{-1}$ and $10^{-2}$ without DSSS; while with DSSS, it was between $10^{-1}$ and $10^{-3}$. In other words, the use of DSSS has the benefit of further reducing the interference effects caused by the communication channel. As a future work, other communication channel could be examined like multipath Rayleigh fading channel.

\section{REFERENCES}

[1] M. Ilyas and I. Mahgoub, "Handbook of sensor networks: compact wireless and wired sensing systems," CRC press, 2004.

[2] T. Samant, "Analysis and Comparison of SMAC and TMAC Protocol for Energy Efficient Dynamic Topology in Sensor Network," International Journal of Electrical and Computer Engineering, vol. 6, pp. 2331, 2016.

[3] S. Chelbi, et al., "An Unequal Cluster-based Routing Protocol Based on Data Controlling for Wireless Sensor Network," International Journal of Electrical and Computer Engineering, vol. 6, pp. 2403, 2016.

[4] W. Dargie and C. Poellabauer, "Fundamentals of wireless sensor networks: theory and practice," John Wiley \& Sons, 2010.

[5] A. A. Jaber and R. Bicker, "Wireless Fault Detection System for an Industrial Robot Based on Statistical Control Chart," International Journal of Electrical and Computer Engineering, vol. 7, pp. 3421-3435, 2017.

[6] C. Y. Hsieh, "Model Study for Temperature Microchange by WSN Technology," International Journal of Electrical and Computer Engineering, vol. 2, pp. 632-638, 2012.

[7] I. F. Akyildiz and M. C. Vuran, "Wireless sensor networks," vol. 4, John Wiley \& Sons, 2010.

[8] P. Torkzadeh and M. Atarodi, "A Power-Efficient LC Quadrature VCO for RFID, Zigbee and Bluetooth Standards," Advances in Electrical and Computer Engineering, vol. 9, pp. 34-38, 2009.

[9] K. Gorantla and V. V. Mani, "Simulink model for Zigbee transceiver using OQPSK modulation under fading channels," IEEE International Conference on Communications and Signal Processing (ICCSP), Melmaruvathur, pp. 0220-0224, 2015.

[10] S. Farahani, "ZigBee wireless networks and transceivers," newnes, 2011.

[11] M. B. Mantri, et al., "Performance analysis of $2.4 \mathrm{GHz}$ IEEE 802.15 .4 PHY under various fading channels," Emerging Trends in Communication Control Signal Processing \& Computing Applications (C2SPCA), IEEE International Conference, pp. 1-4, 2013.

[12] C. Wang, et al., "ZigBee® network protocols and applications," CRC Press, 2014.

[13] B. K. Muni and S. K. Patra, "Low Bit Error Rate ZigBee Baseband Transceiver Design for IEEE 802.15. 4," First International Conference on Mobile and Embedded Technology, Department of Electronics and Communication Engineering, amity School of Engineering and Technology, NOIDA, 2013.

[14] A. K. Anwar and L. Lavagno, "Simulink Modeling of the 802.15. 4 Physical Layer for Model-Based Design of Wireless Sensor Networks," IEEE $3^{\text {rd }}$ International Conference on Sensor Technologies and Applications, Greece, pp. 38-42, 2009.

[15] K. S. a. I. J. M. Alnuaimi, "Performance Evaluation of IEEE 802.15.4 Physical Layer Using MatLab/Simulink," Innovations in Information Technology, Dubai, pp. 1-5, 2006.

[16] R. Ahmad, et al., "Implementation of IEEE 802.15. 4-based OQPSK-pulse-shaping block on FPGA," Computer Applications and Industrial Electronics (ICCAIE), International Conference, IEEE, pp. 459-464, 2011.

[17] P. Mishra and S. Mane, "Implementation of QPSK Modulation on MATLAB Simulation," International Journal of Engineering and Innovative Technology (IJEIT), vol/issue: 5(8), 2016.

[18] P. K. Agarwal, "Design Transceiver for IEEE 802.15. 4 using ZigBee Technology and Matlab/Simulink," International Journal of Science and Research (IJSR), vol. 3, pp. 1901-1906, 2014.

[19] T. D. Memon, et al., "Quadrature phase shift keying modulator \& demodulator for wireless modem," Computer, Control and Communication, 2nd International Conference, Karachi, IEEE, pp. 1-6, 2009.

[20] H. S. Hamid, et al., "Analyze BER Performance of Wireless FSK System," Microwaves and RF, vol. 48, 2009.

[21] L. W. Couch, et al., "Digital and analog communication systems," vol. 6, Prentice Hall, 1997.

[22] N. V. Panicker and A. K. Sukesh, "BER Performance Evaluation of Different Digital Modulation Schemes for Biomedical Signal Transceivers under AWGN and Fading Channel Conditions," International Journal of Engineering and Advanced Technology (IJEAT), vol/issue: 3(5), 2014.

[23] S. K. Kaul, "QPSK, OQPSK, CPM Probability Of Error for AWGN and Flat Fading Channels," Wireless Communication Technologies, Spring, 2005.

[24] R. Kanna, "Design of ZigBee transceiver for IEEE 802.15.4 using Matlab/Simulink," Master Thesis, Dept. Elec. Comm. Eng., National Institute of Technology, India, 2011. 